\title{
A IMPORTÂNCIA DO MÉTODO CANGURU PARA RECÉM-NASCIDOS PREMATUROS E/OU DE BAIXO PESO AO NASCER
}

\author{
Adila Marcela Lima Nunes ${ }^{1}$
}

RESUMO: O número crescente de nascimentos de recém-nascidos prematuros e/ou de baixo peso ao nascer, com considerável taxa de mortalidade destes bebês no primeiro ano de vida, fez com que se pensasse em métodos que objetivassem a redução destes casos, tendo como destaque o Método Canguru (MC). Este vem atraindo a atenção dos estudiosos e profissionais pelo mundo nos últimos anos, por ser um método acessível, de baixo custo e de fácil manipulação, além de apresentar muitos benefícios. Assim, este artigo tem por objetivo denotar a importância do MC para recém-nascidos prematuros e/ou de baixo peso ao nascer e como justificativa demonstrar a importância do Método Canguru para recém-nascidos prematuros e/ou de baixo peso, não como método substitutivo e sim como método somatório às tecnologias clássicas. Realizou-se uma pesquisa bibliográfica, de caráter exploratória, através de trabalhos já publicados, artigos de periódicos, artigos científicos e textos nas bases de dados Scielo, Interfisio, disponibilizados em meio eletrônico, considerando as contribuições dos autores Brasil, Marques, Ribeiro, Silva, Maia, Lamy, com o intuito de enfatizar a importância do MC para o desenvolvimento neuropsicomotor de bebês prematuros e de baixo peso. Portanto, se faz necessário o desenvolvimento e elaboração de parâmetros que atendam aos objetivos do $\mathrm{MC}$, com foco na humanização do cuidado ao $\mathrm{RN}$ e seus familiares, visto que esta prática apresenta muitos benefícios a um custo totalmente acessível.

Palavras-chaves: Recém-nascidos. Mortalidade. Método Canguru.

\section{INTRODUÇÃO}

A elevada taxa de nascimentos de recém-nascidos prematuros e de baixo peso ao nascer constitui um importante problema de saúde pública, visto que uma parte considerável destes bebês morrem antes do primeiro ano de vida. Desta forma, torna-se primordial uma assistência qualificada e a utilização de métodos que visem reduzir a morbimortalidade no período neonatal, tendo como destaque o Método Canguru (MC). Este método engloba quesitos relacionados aos cuidados com o recém-nascido (manejo, atenção às necessidades individuais, cuidados com a dor, som, luz): o amparo à família; a promoção do vínculo mãe/pai/bebê e do aleitamento materno (AM); além do

\footnotetext{
${ }^{\mathrm{I}}$ Pós-graduação em Enfermagem Ginecológica e Obstétrica pela Faculdade Única de Ipatinga. Pós-graduação em Enfermagem em UTI Neonatal e Pediátrica pelo Centro Universitário FAVENI. Bacharel em Enfermagem pela Universidade Estadual do Maranhão. E-mail: adilamarcela@hotmail.com.
} 
acompanhamento ambulatorial após a alta. Sendo assim, o presente trabalho tem como objetivo denotar a importância do MC para recém-nascidos prematuros e/ou de baixo peso ao nascer.

Nesta perspectiva, construiu-se questões que nortearam este trabalho:

- $\quad$ Como se encontra a saúde do RN no Brasil?

- Qual a classificação do recém-nascido prematuro e de baixo peso?

- $\quad$ O que é o MC?

- Quais os benefícios deste método?

Tendo como objeto de estudo os recém-nascidos prematuros e/ou de baixo peso ao nascer, o seguinte problema norteou a pesquisa: Qual a importância do MC para a sobrevida e/ou desenvolvimento destes recém-nascidos?

O MC é um excelente modelo de assistência ao recém-nascido prematuro e/ou de baixo peso e sua família. Ele visa favorecer o vínculo entre a família e o RN, diminuir o tempo de internação na Unidade de Terapia Intensiva (UTI) e/ou unidade hospitalar, estimular o aleitamento materno, reduzir os níveis de estresse e dor, além de outros benefícios para o bom desenvolvimento do bebê.

Diante do exposto, considera-se de imensa relevância esta pesquisa, visto que a introdução precoce deste método poderá ter grande êxito no processo de sobrevida destes recém-nascidos e nos cuidados pelos seus familiares. É importante frisar que este método não substitui as unidades de terapia intensiva neonatal, uma vez que determinados casos tem suas indicações já pré-estabelecidas. Além do mais, ele não visa economizar recursos humanos e/ou técnicos, mas garantir a atenção perinatal e humanizada.

Este trabalho justifica-se em demonstrar a importância do Método Canguru para recém-nascidos prematuros e/ou de baixo peso, não como método substitutivo e sim como método somatório às tecnologias clássicas. Portanto, este deve ser inserido desde à atenção básica, numa fase anterior ao nascimento de bebês prematuros e/ou de baixo peso, através da identificação de gestantes de alto risco para esta ocorrência.

Para alcançar o objetivo proposto, utilizou-se como metodologia a pesquisa bibliográfica, com natureza exploratória, através de trabalhos já publicados, artigos de periódicos, artigos científicos e textos nas bases de dados Scielo, Interfisio, disponibilizados em meio eletrônico. Autores como Brasil, Marques, Ribeiro, Silva, Maia et al, Lamy et al tiveram suas ideias e concepções mencionadas neste estudo. 


\title{
I DESENVOLVIMENTO
}

Anualmente, nascem cerca de 20 milhões de crianças prematuras e/ou de baixo peso, sendo que um terço delas morrem antes de completar o primeiro ano de vida (BRASIL, 2009 apud SILVA, 2014). Segundo Marques (2020), “a Organização Mundial de Saúde (OMS) define o neonato com baixo peso ao nascer como todo nascido vivo com peso inferior a 2,500 gramas, e a prematuridade como o nascimento que ocorre antes da $37^{\text {a }}$ semana de gestação". Além disto, no requisito baixo peso, conforme Neves (2010) apud Maia et al (2011, p. 232), "são incluídos tanto os prematuros quanto os recém-nascidos a termo com retardo no crescimento intrauterino".

\begin{abstract}
A classificação dos prematuros considera a idade gestacional, o peso ao nascer e/ou relação entre idade gestacional e o peso. Utilizando o peso como parâmetro, o prematuro pode ser classificado como de baixo peso ao nascer (RNBP) que tem peso entre 1.501 e 2.50o gramas; recém-nascido (a) de muito baixo peso (RNMBP) com peso entre r.ooo e I.50o gramas e recém-nascido (a) de muitíssimo baixo peso (RNMMBP) com peso inferior a r.ooo gramas (MARGOTO, MOREIRA, 2oII apud SILVA, 2014 p. 17).
\end{abstract}

A atenção à saúde do recém-nascido (RN) é imprescindível para a redução da mortalidade infantil, ainda elevada no Brasil, assim como a promoção de melhor qualidade de vida e a diminuição nas taxas de internações hospitalares. Para França e Lansky (2009) apud Brasil (2014, p. 19), "a maior parte das mortes infantis ocorre nos primeiros dias de vida da criança, e por causas consideradas evitáveis, como infecção, asfixia ao nascer e complicações da prematuridade". Neste contexto, é notória a necessidade de uma assistência qualificada e/ou a utilização de métodos que visem reduzir a morbimortalidade neste período, tendo como ênfase o Método Canguru (MC).

\footnotetext{
O número elevado de nascimentos prematuros e de baixo peso e a necessidade de suporte aos (às) profissionais de saúde conduziram o Ministério da Saúde a apoiar e padronizar ações nas unidades neonatais, lançando em 05/o7/2000, a Norma de Atenção Humanizada ao (a) RN de Baixo Peso. Além dessa norma, tem relevância o Método Canguru-MC, que visa a contribuir para mudanças na postura profissional, com vista à humanização da assistência ao (a) RN (BRASIL, 2011 apud SILVA 2014, p, 13).
}

Este método é essencial na sobrevida e no desenvolvimento do recém-nascido prematuro e/ou de baixo peso, porém não substitui as internações nas Unidades de Terapia Intensiva, tampouco as tecnologias clássicas. É indispensável sua utilização desde o período perinatal, associada a uma adequada assistência dos profissionais de saúde no cuidado obstétrico e neonatal. De acordo com Ribeiro (2020), "é uma alternativa que foi 
criada pelo pediatra Edgar Rey Sanabria em 1979 em Bogotá na Colômbia, para reduzir o tempo de internação e incentivar o aleitamento dos recém-nascidos de baixo peso."

Devida a estas circunstâncias, o MC foi inserido em algumas unidades de saúde brasileiras na década de 90 , sendo incorporado às políticas de saúde no âmbito perinatal. (BRASIL, 2013). No Brasil, este método já vinha sendo utilizado anteriormente, desde I99I, pelo Hospital Guilherme Álvaro em Santos/SP nas enfermarias do Alojamento Conjunto. A partir daí, alguns hospitais brasileiros passaram a fixar normas sobre o uso da posição canguru para a população de mães e bebês prematuros, sem parâmetros bem estabelecidos. (BRASIL 2013). Desde então, a prática de colocar o recém-nascido prematuro ou de baixo peso contra o tórax seja da mãe, do pai ou de qualquer outro familiar ganhou notoriedade no mundo inteiro, ganhando tanto admiradores quanto oponentes, como é de se esperar em todo processo de adoção de novas medidas cientificas.

Nos primeiros is anos de implantação, o MC ganhou abrangência nacional e reconhecimento internacional. Conforme Brasil (2017, p. 13), "O caráter de política pública, o compromisso com as melhores práticas clínicas e as evidências científicas e a integralidade na abordagem do recém-nascido e sua família foram fatores centrais nesse processo".

\begin{abstract}
Em 2015, a Política Nacional de Atenção Integral à Saúde da Criança (Pnaisc) foi instituída pela Portaria GM/MS no 1.130, de 5 de agosto de 2015. Fruto de um extenso trabalho de acúmulo, reflexão, discussão e articulação da CGSCAM com inúmeros e diversos parceiros, a Pnaisc tem como objetivos centrais promover e proteger a saúde da criança e o aleitamento materno, mediante a atenção e os cuidados integrais e integrados da gestação aos 9 nove anos de vida, com especial atenção à primeira infância e às populações de maior vulnerabilidade, visando à redução da morbimortalidade e um ambiente facilitador à vida com condições dignas de existência e pleno desenvolvimento A identificação dos princípios orientadores da Pnaisc (direito à vida e à saúde; prioridade absoluta da criança; acesso universal à saúde; integralidade do cuidado; equidade em saúde; ambiente facilitador à vida; humanização da atenção e gestão participativa e controle social) nos permite, de imediato, compreender sua proximidade em relação aos pilares do MC no Brasil. (BRASIL, 2017, p. 20)
\end{abstract}

O MC é um exemplo de auxílio que se inicia desde o período perinatal, com ênfase na qualidade do cuidado ao RN de forma humanizada, objetivando o bom desenvolvimento do bebê. Vem atraindo a atenção dos estudiosos e profissionais pelo mundo nos últimos anos, e por ser um método de baixo custo e de fácil manipulação possui muitos benefícios, dentre os quais, segundo Brasil (2017):

- Diminui o tempo de separação mãe/pai-filho;

Auxilia para o vínculo afetivo mãe/pai-filho; 


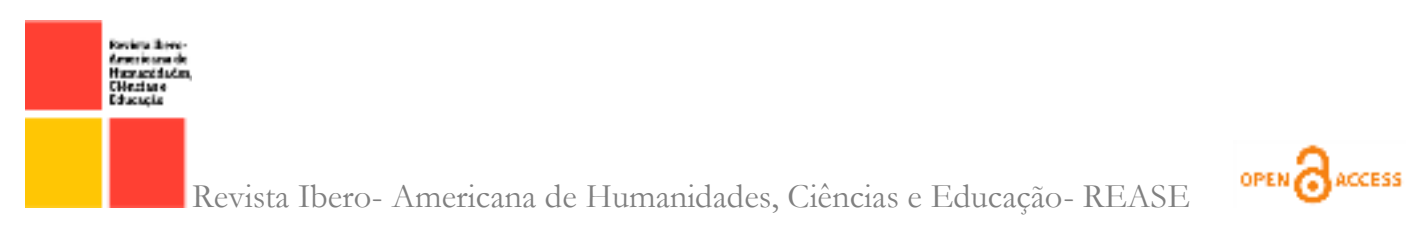

Proporciona maior capacidade e confiança dos pais no cuidado do seu filho, até mesmo após a alta hospitalar;

- Incentiva o aleitamento materno, permitindo maior frequência, precocidade e duração;

Propicia ao recém-nascido melhor controle térmico;

- Colabora para a diminuição do risco de infecção hospitalar;

- Restringi o estresse e a dor;

- Possibilita melhor relacionamento da família com a equipe de Saúde;

- Beneficia ao recém-nascido uma estimulação sensorial protetora em relação ao seu desenvolvimento integral;

Aumenta a qualidade do desenvolvimento neuropsicomotor.

Além dessas vantagens, é de extrema importância que a mãe, pai e/ou qualquer outro familiar que se responsabilize pelos cuidados do $\mathrm{RN}$, recebam instruções dos profissionais de saúde sobre sua importante atuação no cuidado do bebê, ao contribuir com o seu desenvolvimento biopsicossocial, possibilitando a formação e o fortalecimento do vínculo afetivo entre o $\mathrm{RN}$, a mãe, o pai e a família. Para isto, o método deve contar com uma equipe multidisciplinar, que deverá ser composta por médico pediatra e/ou neonatologista, enfermeiro, técnico de enfermagem, fisioterapeuta, terapeuta ocupacional, assistente social, fonoaudiólogo, nutricionista, entre outros.

Embora os avanços e fortalecimento do MC, já comprovados em publicações científicas e notas técnicas, fossem incontestáveis, a garantia existente de que cada recémnascido prematuro, procedente de maternidade brasileiras, tivesse acesso ao preconizado nas três etapas do MC, ainda se mantém como um objetivo a ser almejado em nosso país (BRASIL, 2017).

O método canguru é desenvolvido em três etapas: Primeira etapa: refere-se ao período de internação na terapia neonatal. No local da terapia, a família pode receber informações e é preparada para aderir ao mesmo, desenvolvendo o método Mãe Canguru parcialmente; Segunda etapa: sobre o período em que o neonato, clinicamente estável e com mais de $\mathrm{r} .250 \mathrm{~g}$, permanece com a mãe na enfermaria Mãe Canguru de estabilização, desde que ela esteja pronta para recebê-lo; Terceira etapa: é a fase de acompanhamento ambulatorial para a vigilância do crescimento e desenvolvimento do $R N$, interação família-bebê e detecção precoce de situações de risco. (VENANCIO 2004, apud MAIA et al 2011, p. 232)

É importante salientar que cada etapa do método tem o objetivo de oferecer à mãe e/ou pai condições para a ampliação do vínculo, proporcionando sua qualificação para o 
cuidado com o seu filho, o que favorecerá para uma melhor adequação da família à UTI, com repercussão na melhoria das condições de saúde e desenvolvimento da criança.

A partir da Portaria ${ }^{\circ}$ 930, as unidades neonatais no âmbito do SUS passam a ser divididas de acordo com as necessidades do cuidado, nos seguintes termos: I Unidade de Terapia Intensiva Neonatal (UTI neonatal). II - Unidade de Cuidado Intermediário Neonatal (Ucin), com duas tipologias: a) Unidade de Cuidado Intermediário Neonatal Convencional (UCINCo). b) Unidade de Cuidado Intermediário Neonatal Canguru (UCINCa). (BRASIL, 2017 p. 19)

Ressalta-se que, se nesta portaria a conhecida segunda etapa do MC recebe em sua designação o termo canguru, não significa dizer que apenas aqui se abrangem tais cuidados. Estes já devem ser instaurados desde o pré-natal e transcorrer por toda a internação. (BRASIL, 2017).

Em vista disto, nota-se o Método Canguru como fator importante para o desenvolvimento e sobrevida de recém-nascidos prematuros e/ou de baixo peso, tanto que o Ministério da Saúde o admitiu como uma Política Nacional de Saúde, introduzindo-o no contexto da humanização da assistência neonatal e servindo como meio para atenuar os efeitos negativos da internação neonatal sobre os bebês e suas famílias. Sendo assim, foi divulgada, em dezembro de 1999, por meio da Portaria nº 639 GM/MS, a Norma de

Atenção Humanizada ao Recém-Nascido de Baixo Peso - Método Canguru. (LAMY et al, 2005)

Para compreendermos a trajetória do Método Canguru como Política Nacional de Saúde, no Brasil, é necessário destacar que a humanização no ambiente da Unidade de Terapia Intensiva Neonatal (UTIN) é uma questão de importância crescente na qualidade da atenção ao recém-nascido. Portanto, humanização e qualidade da assistência não podem ser vistas como fenômenos dissociados. Essa ampliação do olhar sobre o método foi de fundamental importância para a sua disseminação no Brasil. Entretanto, outros dois fenômenos também estão inseridos nesse contexto. Primeiro, o crescente reconhecimento, por parte das equipes de neonatologia, da importância dos cuidados maternos para a recuperação dos bebês e, em segundo lugar, o momento atual no qual a humanização da assistência tem sido apresentada como política nacional do Ministério da Saúde. (LAMY et al, 2005, p. 660)

O pontapé inicial sobre o Método Canguru - origem, descrição, benefícios e adversidades - conduziu a ideia da sua importância para a assistência neonatal e ao desenvolvimento de uma recomendação de utilização nacional, que não deveria ser considerada uma técnica qualquer, todavia num projeto contextualizado em uma sugestão mais ampla de humanização. 


\section{CONSIDERAÇÕES FINAIS}

Cada vez mais, estudos nas áreas de neonatologia e obstetrícia vêm colaborando para a sobrevivência de recém-nascidos prematuros e/ou com BPN e diminuindo os níveis de mortalidade infantil, à medida que estes recebam assistência médica especializada imediatamente logo após o nascimento, e em local adequado para acolher este bebê de acordo com a sua necessidade. Além disto, incertezas sobre a qualidade de vida e impactos sobre o crescimento e desenvolvimento destes bebês, vem estimulando interesse e cuidado ao prognóstico desta população, uma vez que metade destes prematuros apresenta alteração no desenvolvimento sensorial e neurológico.

Diante disto, o Método Canguru tornou-se um exemplo de modelo que está se dispersando por todo o mundo. É extremamente importante para o bom desenvolvimento neuropsicomotor do bebê, desde o período perinatal até a alta hospitalar, e muito eficaz por ser de baixo custo. Ele reduz os índices de morbimortalidade infantil, que tem alta taxas no primeiro ano de vida, e tendo como causas principais a prematuridade e o baixo peso ao nascer. Além disto, como demonstrado neste estudo, é uma técnica muito eficaz, pois além

de fortalecer o vínculo entre mãe e bebê - não deixando de mencionar a importância da 406 participação do pai e/ou de outros familiares em todo o processo - também incentiva o aleitamento materno exclusivo, reduzindo o tempo de internação hospitalar e possíveis complicações a saúde e desenvolvimento do RN.

Apesar de ser essencial na sobrevida e no desenvolvimento do recém-nascido prematuro e/ou de baixo peso, não substitui as internações nas Unidades de Terapia Intensiva, tampouco as tecnologias clássicas. É indispensável sua utilização desde o período perinatal, associada a uma adequada assistência dos profissionais de saúde no cuidado obstétrico e neonatal. No entanto, isto ainda constitui uma adversidade, pois o mesmo requer a participação de todo uma equipe multidisciplinar, além de uma educação continuada, o que nem sempre podemos contar nos hospitais públicos do nosso país. Lamy et al (2005, p. 666), reitera “a importância de estudos que avaliem a implantação da Atenção Humanizada ao Recém-Nascido de Baixo Peso e seu impacto nos resultados neonatais em nosso país”.

Em vista disso, acredita-se ser necessário o desenvolvimento e elaboração de parâmetros que atendam aos objetivos do $\mathrm{MC}$, com foco na humanização do cuidado ao RN e seus familiares, visto que esta prática apresenta muitos benefícios a um custo 
totalmente acessível. Além do mais, sua importância para a assistência neonatal deveria ser considerada como um modelo nacional de utilização.

\section{REFERÊNCIAS}

BRASIL. Ministério da Saúde. Secretaria de Atenção à Saúde. Departamento de Ações Programáticas Estratégicas. Atenção humanizada ao recém-nascido de baixo peso: Método Canguru. 2 ed. Brasília: Editora do Ministério da Saúde, 2013. 204 p. Acesso em 25 de março de 202I, disponível em:https://bvsms.saude.gov.br/bvs/ metodo_canguru_manual_zed.pdf

BRASIL. Ministério da Saúde. Secretaria de Atenção à Saúde. Departamento de Ações Programáticas Estratégicas. Atenção à saúde do recém-nascido: guia para os profissionais de saúde. 2 ed. Brasília: Editora do Ministério da Saúde, 2014. 192 p. Acesso em 28 de março de 202I, disponível em: https://bvsms.saude.gov.br/bvs/publicacoes/atencao_saude_r -vi.pdf

BRASIL. Ministério da Saúde. Secretaria de Atenção à Saúde. Departamento de Ações Programáticas Estratégicas. Atenção humanizada ao recém-nascido: Método Canguru: manual técnico. 3 ed. Brasília: Editora do Ministério da Saúde, 2017. 34op. Acesso em 22 de março de 2021, disponível em: file://C:/Users/pc/Downloads/atencao_humanizada _manual_zed.pdf

LAMY, Zeni Carvalho et al. Atenção humanizada ao recém-nascido de baixo peso 407

Método Canguru: a proposta brasileira. Ciência \& Saúde Coletiva, 2005. 1o (3) p. 659 - 668. Acesso em or/o5/2021, disponível em: file:///C:/Users/pc/Downloads/7ZP4gr3Pp.pdf

MAIA, Jair Alves et al. Método Canguru: a importância da família na recuperação do recém-nascido de baixo peso. Enfermagem em Foco 2011; 2(4):231-234. Acesso em 20 de março de 202I, disponível em: file://C:/Users/pc/Downloads/ \%20CANGURU.pdf

MARQUES, Marcella. Benefícios do método canguru no tratamento de prematuros de baixo peso ao nascimento. 2020. Acesso em 20 de março de 2021, disponível em: https://interfisio.com.br/beneficios-do-metodo-canguru-no-tratamento-de-prematurosnascimento/\#: :text=A\%2oliteratura\%2oevidenciou\%2odiversas\%2ocontribui\% $\mathrm{C}_{3} \% \mathrm{~A} 7 \% \mathrm{C}$ 3\%Bses,no\%2osistema\%2oneurol\% $\mathrm{C}_{3} \% \mathrm{~B}_{3}$ gico\%3B\%2omelhora\%2ono

RIBEIRO, Sani Santos. Método canguru: o que é e como fazer. 2020. Acesso em 25 de março de 202I, disponível em: https://www.tuasaude.com/metodo-canguru/

SILVA, Joise Magarão Queiroz. Significado para mães sobre a vivência no método canguru. Salvador: 2014, 79 p. Acesso em: 02 de abril de 2021, disponível em: file:///C:/Users/pc/Downloads/332_-_dissertacao_-josise_magarao_queiroz_silva.pdf 